San Jose State University

SJSU ScholarWorks

Faculty Publications

Health Science and Recreation

January 2007

\title{
Responsibility for HIV Prevention: Patterns of Attribution Among HIV-seropositive Gay and Bisexual Men
}

C. Offer

O. Grinstead

E. Goldstein

Edward M. Mamary

San Jose State University, edward.mamary@sjsu.edu

N. Alvarado

See next page for additional authors

Follow this and additional works at: https://scholarworks.sjsu.edu/healthsci_rec_pub

Part of the Public Health Commons

\section{Recommended Citation}

C. Offer, O. Grinstead, E. Goldstein, Edward M. Mamary, N. Alvarado, J. Euren, and W. Woods. "Responsibility for HIV Prevention: Patterns of Attribution Among HIV-seropositive Gay and Bisexual Men" AIDS Education and Prevention (2007): 24-35.

This Article is brought to you for free and open access by the Health Science and Recreation at SJSU

ScholarWorks. It has been accepted for inclusion in Faculty Publications by an authorized administrator of SJSU

ScholarWorks. For more information, please contact scholarworks@sjsu.edu. 
Authors

C. Offer, O. Grinstead, E. Goldstein, Edward M. Mamary, N. Alvarado, J. Euren, and W. Woods 


\title{
RESPONSIBILITY FOR HIV PREVENTION: PATTERNS OF ATTRIBUTION AMONG HIV-SEROPOSITIVE GAY AND BISEXUAL MEN
}

\author{
Claudine Offer, Olga Grinstead, Ellen Goldstein, \\ Edward Mamary, Nicholas Alvarado, Jason Euren, \\ and William J. Woods
}

\begin{abstract}
The Seroconversion Narratives for AIDS Prevention (SNAP) study elicited narratives from recently infected seropositive gay and bisexual men that described the circumstances of their own seroconversion. This analysis of the narratives explored participants' attributions of responsibility for HIV prevention before and after they became infected. Before becoming infected with HIV, responsibility for prevention was often attributed to HIV-negative individuals themselves. These retrospective attributions revealed themes that included feelings of negligence, a sense of consequences, followed by regret. After seroconversion, responsibility for HIV prevention was primarily attributed to HIV-positive individuals themselves. Themes within these attributions included pledges to avoid HIV transmission, a strong sense of burden related to the possibility of infecting someone, and risk reduction strategies that they implemented in an attempt to avoid HIV transmission. Greater understanding of ideas related to responsibility has the potential to increase the effectiveness of HIV prevention interventions.
\end{abstract}

Although discourse about HIV-positive individuals and personal responsibility emerged years ago (Bayer, 1996), there was an understandable reluctance to address prevention with HIV-positive individuals in order to avoid blaming or further stigmatizing this vulnerable population. However, in their current principles of HIV prevention, the National Association of People With AIDS (NAPWA) promotes the importance of addressing people living with HIV/AIDS in the development of successful HIV prevention strategies (NAPWA, 2005). NAPWA advocates for the involvement of people living with HIV in creating those strategies. Advancing HIV

Claudine Offer, Olga Grinstead, Ellen Goldstein, Nicholas Alvarado, Jason Euren, and William J. Woods are with the Center for AIDS Prevention Studies, University of California, San Francisco. Edward Mamary is with San Jose State University, San Jose, CA.

The SNAP study was supported by the Universitywide AIDS Research Program (UARP) at the University of California, Office of the President (Olga Grinstead, principal investigator). The authors express their gratitude to the SNAP participants.

Address correspondence to Claudine Offer, MPH, Center for AIDS Prevention Studies, University of California San Francisco, 50 Beale St., Suite 1300, San Francisco, CA 94105; e-mail: Claudine.Offer@ucsf.edu 
Prevention (AHP), a recent Centers for Disease Control and Prevention (CDC) initiative, also called for an increased focus on prevention with HIV-positive individuals (CDC, 2003).

With the prioritizing of HIV-positive individuals in prevention programs, there has been a growing call to gain a better understanding of risk behavior among HIV-positive persons (Crepaz, Hart, \& Marks, 2004; Crepaz \& Marks, 2002) and to develop relevant prevention strategies that address the specific risk-reduction challenges of those living with HIV (Gordon, Forsyth, Stall, \& Cheever, 2005). Most people who are aware of their HIV-positive status wish to avoid transmitting the virus (Marks, Burris, \& Peterman, 1999). Despite these findings, HIV-positive men who have sex with men (MSM) have reported unprotected anal sex with partners of negative or unknown status in several studies (Chen et al., 2002; Colfax et al., 2001; Weinhardt et al., 2004). Meanwhile, HIV prevention interventions for people living with HIV have been successful in reducing risk behaviors (Crepaz et al., 2006; Johnson, Carey, Chaudoir, \& Reid, 2006).

Gore-Felton et al. (2005) have asserted the need for preventive interventions that address both altruistic and other types of motivations in order to effectively support people living with HIV who face challenges in reducing high-risk behavior. While investigating the feasibility and desirability of directing HIV prevention toward HIV-positive individuals, Kok (1999) recognized the likelihood of personal responsibility as a motivational factor in risk behavior. More recently, in the Seropositive Urban Men's Study (SUMS), participants expressed a belief in their responsibility to protect others (Wolitski \& Bailey, 2005). In the same study, HIV-positive MSM who reported unprotected anal insertive sex perceived less responsibility to protect their partners from HIV than those who did not report unprotected anal insertive sex (Parsons, Halkitis, Wolitski, Gómez \& the Seropositive Urban Men's Study Team, 2003). Furthermore, an association between negative affective states, such as anger or depression, and unprotected anal intercourse with HIV-positive men, appeared to be mediated by attributions of responsibility for protecting sex partners (Marks, Bingman \& Duval, 1998).

Like other motivational factors that include a concern for others, the concept of responsibility and how it is put into practice may have an effect on an HIV-positive individual's HIV transmission risk behavior. A link between an individual's personal theory about their HIV seroconversion event and their current risk behavior is supported by several studies in which attributions of blame for infection (Bingman, Marks, \& Crepaz, 2001) and attributions of responsibility for protection (Marks et al., 1998) were both found to be associated with HIV transmission risk behavior among men living with HIV. Additional exploration of the attribution of responsibility for HIV prevention is one way to gain further understanding of the concept of responsibility and discover ways that it may be involved in supporting a reduction of risk behavior.

The Seroconversion Narratives for AIDS Prevention (SNAP) study elicited narratives from recently infected seropositive gay and bisexual men that described the circumstances of their own seroconversion in order to determine if individual, interpersonal and structural attributions in these narratives were related to participants' post-infection prevention strategies. (Grinstead, 2006; Grinstead, Alvarado, Goldstein, Woods, \& Euren, 2005). During the initial analysis of the SNAP findings, a strong theme of responsibility emerged. In nearly all of the interviews, participants made direct and spontaneous mention of responsibility or clearly implied ideas about 
responsibility. This analysis of the SNAP interviews further explored the participants' attributions of responsibility for HIV prevention, both before and after their seroconversion event.

\section{METHODS}

\section{PARTICIPANTS}

The SNAP study recruited 28 participants from community-based AIDS service organizations (ASOs) and by word of mouth in San Francisco. Recruitment took place in agencies that provided services for HIV-positive gay and bisexual men with an intention to recruit an ethnically diverse sample that represented people living with HIV/AIDS in San Francisco. Eligibility was limited to men who identified as gay or bisexual, who were over 18 years of age, who believed they had been infected with HIV in the 2 years prior to their participation, and who could be interviewed in English.

\section{PROCEDURES}

Information about the study was provided to agencies and their clients in the form of flyers and full-page posters that provided a contact number for further inquiry. Agency staff and those who had participated in the study also referred potential participants. Men who expressed interest in participating in the study were screened for eligibility and provided informed consent. Interviews lasted for 1 to 1.5 hours, after which participants were given referrals as needed and provided with a $\$ 50$ reimbursement for their time. All SNAP study procedures were reviewed and approved by the University of California, San Francisco Committee on Human Research.

\section{INTERVIEW INSTRUMENT}

The SNAP study used semistructured qualitative interviews based on a narrative approach. These narratives provide the participants' perception of themselves, their environment and the nature of their HIV seroconversion that is ideal for the purpose of this thematic analysis. The interview guide (shown in Table 1) included two major sections: (a) the seroconversion narrative (each participant's detailed story about how he believes that he became infected with HIV) and (b) current prevention practices (what each participant does, if anything, to avoid transmitting HIV to others). To elicit the seroconversion narrative, the interviewer began by asking the respondent, "Tell me the story about how it happened that you were infected with HIV." The interviewer then allowed the narrative to emerge before probing. Additional sections focused on risk behaviors and risk predictors as an HIV-positive man. Questions and probes did not refer to responsibility; any reference to responsibility was spontaneous. Interviews were audiotaped and transcribed verbatim.

\section{DATA ANALYSIS}

Interviews from 26 participants were included in this thematic analysis; two interviews were excluded from this analysis. One interview was an entirely needle related narrative by a man who did not identify as gay/bisexual. In the other interview, the participant revealed that he had most likely seroconverted 10 years prior instead of within the last 2 years. During the first reading, the investigator sought only to become familiar with the overall narrative and context of the seroconversion event. Areas of text that addressed responsibility were noted and a log was kept to define specific aspects of the concept of responsibility focusing on participants' ideas about responsibility for HIV transmission risk-reduction. These ideas were explored based 
TABLE 1. SNAP Interview Guide

Part I: Seroconversion Narrative

1. Tell me the story about how it happened that you were infected with HIV.

2. When did you first test positive for HIV?

Part II: Current Prevention Practices

1. What does your life look like now since you became infected with HIV?

2. How do you express yourself sexually now?

3. Tell me about the last time you had sex where you were confident that there was no risk of transmitting HIV.

4. How about the most recent time you had sex where you thought there might have been some risk to transmit HIV?

5. Have you changed anything about the ways that you use drugs or alcohol since you were infected with HIV? 5a. (Ask only injection drug users) What about needle use?

5b. (Ask only injection drug users) Tell me about the last time you injected drugs around other people where you were confident that there was no risk of transmitting HIV through needle use?

5 c. How about the most recent time injected drugs where you thought there might have been some risk to transmit HIV?

6. What message would you give to a gay/bisexual man who is HIV+ and wants to avoid transmitting the virus to others? What would you tell that person to do?

7. What message would you give to someone who is HIV- and wants to avoid getting HIV?

8. Is there anything I didn't ask you that you would like to tell me about? Anything that is important for me to better understand your story?

on the following criteria: (a) that participants directly mentioned responsibility for risk reduction behaviors or (b) that they implied the assignment of responsibility by using words such as blame or should.

The second reading of the narratives focused on the sections addressing responsibility within the context of the entire interview. After this second reading, two brief summaries of each narrative were written. The first described the participant, his seroconversion narrative, and themes of responsibility both before and around the time that he believed he became infected. The second summary focused on the participant's current feelings about HIV prevention, his sexual risk reduction behaviors and the roles of both HIV-negative and HIV-positive partners in HIV prevention. Finally, the attribution of responsibility was coded for each interview. Criteria for assigning attribution included the clarity and strength with which statements were made and the number of statements in which attribution was made. Attribution for responsibility before and after seroconversion was coded as attribution of responsibility to HIV-positive individuals, HIV-negative individuals, or both. These categories were mutually exclusive. Finally, quotes demonstrating attributions were reviewed and coded for themes.

\section{RESULTS}

SAMPLE CHARACTERISTICS

The 26 interviews used in this thematic analysis were collected between February and June 2003. Eighteen participants $(69 \%)$ reported being infected for less than 2 years and $22(85 \%)$ less than 3 years $(m=2.4$ years, range $=1-7$ years). Eleven $(42 \%)$ identified as Caucasian, 9 (35\%) as African American, 4 (15\%) as Latino, $1(4 \%)$ as Asian/Pacific Islander, and $1(4 \%)$ as Native American/Caucasian. The average age of the participants was 33 years, with an age range of 19 to 44 years. One participant was in his teens $(4 \%), 5$ participants were in their 20s $(19 \%), 14$ were in their 30 s $(54 \%)$, and 6 were in their 40 s $(23 \%)$. Twenty-one of the 26 participants $(81 \%)$ reported using one or more substances at the time of they believed they were infected. Of these, $48 \%$ reported using speed and $19 \%$ reported alcohol. Other substances reportedly used during a participant's seroconversion event included: cocaine, crack, marijuana, 
ecstasy and poppers. Three of the 26 participants were in what they described as a committed relationship (boyfriend, significant other) at the time of their seroconversion event.

\section{ATTRIBUTIONS BEFORE SEROCONVERSION}

When the participants reflected on their sexual practices before seroconversion, attributions emerged that placed responsibility for preventing HIV transmission on (a) HIV-negative people, (b) both HIV-negative and HIV-positive people, or (c) HIV-positive people.

Before Seroconversion: Attribution of Responsibility to HIV-Negative People. Eleven of the participants indicated that they felt HIV-negative men were primarily responsible for preventing HIV infection. Some addressed responsibility directly whereas others implied it through their ideas about prevention strategies or by their expression of feelings of anger or blame toward themselves. Within these attributions, several themes emerged: a sense of their own risk taking, the consequences, and feelings of regret.

Some of the participants made statements that revealed their own sense of risk taking, prior to seroconversion, implying that they were responsible for their own HIV infection. This participant expresses the gamble that he felt he took with his own life. "And I know most of the time I didn't use condoms and I know sometimes I did use condoms. So it was like playing Russian roulette with my life." Other participants described strategies for HIV prevention and risk reduction in their relationships or sexual practices prior to their infection. One participant explained that he had experienced a "lapse" at some point in his safer sex strategy; others expressed the difficulty in balancing the complexities of HIV prevention in their own decision-making process as HIV-negative men.

\footnotetext{
I mean I think too I was progressing into more risky behavior. I'm really not a barebacker person but I was definitely kind of sick of using condoms and I was trying to like balance that whole [HIV prevention] you know. I was trying to make wise decisions based on my dislike of condoms per se. So I would use condoms if I thought something was risky or somebody was HIV-positive for sure. And other times I would just take like educated risks. And I knew I was risking myself. I mean, I had all the information and I chose to go ahead and do it anyway knowing that I potentially would become positive.
}

Participants also expressed a sense of responsibility for their behavior or choices. The following participant stated that he accepted the consequences of his behavior immediately upon learning of his HIV test results: "Well I got myself in this situation. I'm like, 'Now I have to live with the repercussions as far as what I did." Several participants expressed regret for not taking responsibility to prevent HIV infection. "Now-the biggest things that I deal with honestly are still my anger and disappointment in myself of having allowed myself to be-to put myself at risk in that way."

Before Seroconversion: Attribution of Responsibility to Both HIV-Negative and

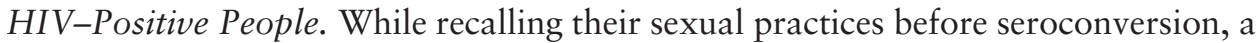
few of the participants expressed the idea that HIV prevention was a shared responsibility between HIV-negative and HIV-positive individuals. Three themes emerged within this set of attributions including commitment, consideration, and experience.

One participant described negotiating HIV prevention strategies with his HIV-positive partner. He explained that his feelings of love and his commitment to 
his partner were factors in his decision-making about risk reduction. He felt that it was not solely his partner's responsibility to protect him from HIV infection. The following quote revealed how this participant reached a mutual decision, with his partner of 3 years, to not use condoms and to implement an alternative harm reduction practice instead:

And he didn't want to come inside of me. As a matter of fact he had a big problem with being inside of me without a condom. You know the first couple times. And when I told him I wasn't worried because I said, "I'm not gonna worry about this." He was somebody that I want to be with and I want to experience life with and love with. And so he would be in me without a condom but he would ejaculate on me as opposed to in me.

One young participant who was infected as a teenager said he didn't blame his partner but, by stating that he felt the partner was inconsiderate and that he felt that he had been taken advantage of, revealed an attribution of some responsibility to his partner as well as to himself:

\begin{abstract}
Like I don't blame the person that infected me; I don't lay any of that on him you know ... I just really think that he is just really inconsiderate. I mean I don't hate him you know ... I don't necessarily have any real feelings towards him. I just really think that ... I mean it's my life you know. You know I made the decision to sneak out of the house and to do all of this stuff you know, to put myself at risk. And you know I put myself out there. I think that you know I was taken advantage of on some level. But at the same time I know that I kinda also put myself out there.
\end{abstract}

Another young participant acknowledged shared decision making while suggesting that his partner should have taken more of the responsibility for preventing HIV infection since his partner had more sexual experience: "I guess I feel upset a little but I feel he maybe should have been more responsible because I was the inexperienced one. But I also recognize that it takes two people to not make the decision that we didn't make or however that works."

Before Seroconversion: Attribution of Responsibility to HIV-Positive People. One participant implied that he held his partner responsible for his infection. In addition to expressing strong feelings of blame and anger, this participant expressed a strong desire to hold his partner legally responsible. He was the only participant to mention the aspect of legal responsibility for HIV transmission.

I thought about killing him, and then I thought I would go and kill myself. Then I thought I don't want to go sit on death row for killing this motherfucker ... I was like, "Can I knowingly have him put in jail for knowingly giving me this shit?" And I thought about that there was a law that's saying that it was a crime to, you know infect someone knowingly with the HIV virus.

Interestingly, the same participant experienced a shift in his thinking about the partner that may have infected him. His initial strong reaction came immediately after receiving the results of his HIV test. Later he allowed his partner the benefit of the doubt: “Then I thought about, 'Maybe he didn't know.' You know I started making excuses. So I got counseling, I talked and it's like, 'This [HIV] is not a death sentence.'”

\title{
ATTRIBUTIONS AFTER SEROCONVERSION
}

The second portion of the interviews reflected the current lives of the participants as well as their HIV prevention ideas and practices. Many of the narratives reveal sto- 
ries of participants still adjusting to their HIV-positive status and exploring relationships, disclosure, sexuality, and HIV prevention strategies. Several participants discussed recovery from drugs and alcohol. Others described their commitment and activism in community HIV prevention efforts. When participants discussed their current perspective as HIV-positive men, they attributed responsibility for preventing HIV transmission to HIV-positive people or both HIV-positive and HIV-negative people. After seroconversion, none of the participants attributed responsibility for HIV prevention solely to the HIV-negative partner.

After Seroconversion: Attribution of Responsibility to HIV-Positive People. After their seroconversion, most participants indicated that they felt HIV-positive men were primarily responsible for preventing HIV infection. In statements that revealed these attributions several themes arose including personal pledges to prevent HIV and a strong sense of burden for HIV prevention. Strategies for HIV risk reduction also surfaced as a theme within attributions made after seroconversion.

Statements such as "I just will not be a person that spreads this" reveal the strength of feelings about responsibility for HIV prevention among HIV-positive individuals and were common in the interviews. The following quote also revealed this participant's strong desires to avoid transmitting HIV: "It's always safe now . . . Knowing that I'm HIV positive I won't put nobody through this."

Some participants anticipated feelings of guilt, remorse, or lack of self-acceptance if they did indeed transmit HIV to a partner. Participants stated that they could not "deal" or "live with" themselves if they infected someone with HIV. These quotes also communicate the strong desire of these participants to avoid transmitting HIV: "Because I care about others ... I just can't live happy and relaxed having it in my head I'm infecting people. I just can't. I can't live with that." Or, "I couldn't live with myself ... if somehow I infected somebody." Another participant revealed a sense of burden that he felt for HIV prevention when he described the stress that comes with feelings of responsibility for HIV-negative partners:

Anybody that is a positive that's dealing with someone that's negative there is a risk. And it actually is the responsibility for that person. It's a responsibility for the positive person . . . I'm not sayin' relationships won't work like that; that's not what I'm sayin'. But I'm sayin' it adds stress or more responsibility to the relationship to where you can be at ease with a person that's already positive.

Many men described risk reduction strategies that revealed their own sense of responsibility for prevention of HIV transmission and their strong desire to avoid infecting their partners. These strategies included selecting specific sexual behaviors (such as oral sex or being the receptive partner during anal sex) partnering only with other HIV-positive people, or choosing celibacy: "I'm being probably extra careful now about oral sex because I don't want my lover to get infected" Another participant stated: "He was a top [anal insertive partner]. Generally that's how I will have it now because I don't want to infect anybody." Several participants discussed strategies such as partnering with other HIV-positive men:

Somebody actually said to me, "I think that it's great that you made that decision to protect you and those that are around you by choosing to have only positive partners." That was something that I really needed to hear. Because what I'd been hearing of course from negative guys is you shouldn't close yourself off to any type of relationship... But in the same instance I also want to protect my community and those that are around me, and I'm not successful wearing condoms. So that means I think its positive guys. 
Although the next participant also made statements reflecting an attribution of shared responsibility, here he discussed his own choice of celibacy as a strategy for HIV prevention:

\begin{abstract}
I don't want to be alone for the rest of my life, and yet, I can never see myself going into another relationship again. Because ... I don't know, things may change in the future, but I could never-because I don't know how it happened, you know. This is my best-case scenario. Because I don't know how it happened I don't want it to happen to anybody else and I will not expose them to it.
\end{abstract}

After Seroconversion: Attribution of Responsibility to Both HIV-Positive and $H I V-N e g a t i v e$ People. Several participants indicated that they now saw the responsibility for HIV prevention as something to be shared by both HIV-positive and HIV-negative individuals. For some, support and assistance from HIV-negative individuals was key. Others felt that HIV-positive individuals should take the lead in HIV prevention whereas others addressed an equal balance between partners in decision making. This participant advised HIV-negative people to consider the burden that those living with HIV experience and to help them carry the responsibility:

\begin{abstract}
I would say talk about it. Just bring it up [HIV status], no matter how-I mean you're negative and you want to stay that way, then bring it up. I mean it's on your shoulders to bring it up. Even though it should be on the other [HIV-positive] person's shoulders to talk about it, just understanding that they got so much fear, they got so much issues, so much - all this stuff going on. But this person who is negative is really in a place of power for them, themselves and realizing that and saying this is my boundary, and just sticking to that and talking about it.
\end{abstract}

This participant agreed that responsibility is shared, but unlike the previous participant, he encouraged those who are HIV-positive to consider taking a larger part of the shared responsibility: "I also believe that it's up to everybody to protect themselves. But somebody who's positive, I think, needs to really hit the high road and avoid, to the best of their ability, from passing it on to anybody else."

Others recognized that two people can be part of a decision-making process about HIV prevention practices. This participant encouraged other HIV-positive individuals to disclose their status so that partners can make informed decisions:

I would tell that person to tell the person they're going to have sex with so the other person has the option of making an informed decision. Because if that person has an informed decision then that way there is no possible risk of transmission. I mean yeah, there is a possibility of transmission. You could be informed and still get it. But it gives the other person the option to decide and to be honest and truthful with your partners.

\title{
SHIFT OF ATTRIBUTION AFTER SEROCONVERSION EVENT
}

When reflecting on the time before they were infected with HIV, most participants attributed responsibility for HIV prevention to HIV-negative individuals. As HIV-positive individuals, however, their attributions of responsibility were primarily to HIV-positive individuals. The following two quotes illustrate one participant's shift of his sense of responsibility.

When I look back [to being HIV-negative] I really can't even remember a face, only because I realize that that was my responsibility ... I absolutely have no bad feelings towards him ... I got myself into that situation. I mean for lack of a better term I asked for that situation. So I actually have no real ill feelings. 
Later in this interview while discussing his current practices he stated:

I'm responsible for myself but I'm at the same time responsible for who I'm with as well. Could I engage with sex with somebody who's partying [using party drugs] or something like that? Probably not. Because it compromises their point of view. It clouds their judgment. And they may be willing to do things that I'm not willing to.

As another example of shifting attributions, this participant acknowledged his responsibility and role in decision making prior to his seroconversion but now feels that it is the full responsibility of the HIV-positive partner, regardless of the decisions of HIV-negative partners, to prevent transmission of HIV.

I don't want to feel like I'm blaming him because I really don't. It was all my decision ... However, that being said ... if he had said to me a year ago, "Look, I don't care what risks you're willing to take, if you're not also positive we're not having unsafe sex" ... I think now that's just bullshit. I will not have unsafe sex with anyone period anyway now! You know I am not gonna pass this around. But God knows there's nobody that's going to say to me now, "That's a risk I'm willing to take," and I'm gonna say, "Oh okay. Bend over." There's no fucking way! . . I'm not gonna give this to somebody else.

\section{OBSTACLES TO HIV PREVENTION}

While examining the seroconversion narratives, several factors emerged as obstacles to the participants' likelihood or ability to include responsibility as a part of their HIV prevention strategy. Even men who had strong intentions to avoid HIV infection were sometimes overwhelmed in that moment and not able to protect themselves for a variety of reasons. We report here on three of the most common themes addressing external obstacles: age and a lack of sexual experience, substance use/addiction, and issues related to survival, such as money and sex work.

Age/Experience. Two participants related how their age or lack of sexual experience influenced their decisions about HIV risk reduction practices during the event in which they believed their seroconversion occurred. In both cases it is apparent that they had entrusted their partners with responsibility regarding HIV prevention.

And I remember giving him like the condom and I think I put it on. But I think it either broke or he took it off or something like that. Like I still allowed him to like-I think I still allowed him to fuck me at the time. I think I told him to put on a condom and I think he expressed that he didn't like to wear 'em. I think he like assured me that he didn't have any like STDs and that he doesn't have anything; he's okay and it's okay to have sex with him. I was, I think, 16 at the time, so I kind of like went a long with him or something, and I was like "whatever." And we played around and had unprotected sex.

As someone with no previous sexual experience the following participant counted on his experienced partner to take the lead:

Then I said, "Well probably, I mean yeah, because I've never had sex at all before." And he said, "Well, I just got tested last October and my results were negative, so I'm safe." And so the third time he just, you know, we were like laying in bed sleeping and he sort of woke up and he just sort of proceeded without [a condom]. And I guess I took my cue from that because he was far more experienced and had been having sex for 10 years already. Hindsight is $20 / 20$ but foolishly I took my cue from the wrong person.

Drug Use/Addiction. Participants described the impact that their drug use had on their ability to protect themselves, such as this man: "Well, basically I was using drugs. And some-times I would use rubbers, sometimes I wouldn't and I don't know. 
It's like when you're doing drugs your head gets fogged." The following participant articulated how he now saw that his drug use prevented him from "thinking responsibly" and making conscious decisions to reduce his risk:

\begin{abstract}
Yeah, it all comes down to just thinking responsibly and the problem with that is when you're that fucked up it just-it just doesn't occur to you to be responsible. It does$n$ 't-it's not a priority. With me it wasn't anyway. It wasn't a priority in my mind. Oftentimes it wasn't-I didn't make a conscious decision not to be safe ... It never entered my mind as an issue. And I don't know how I could have made it to where I did consider those things other than not being impaired at the time and thinking with impaired judgment. So the only solution I saw was you just gotta get a grip and not put yourself in those positions where you'll do that.
\end{abstract}

Money/Survival. Several participants' narratives included stories of sex work as a means of income or survival and how the ability to negotiate safer sex was impeded due to the need for money.

\footnotetext{
Right now I always use condoms-always. But you know what? Sometimes I have problems. Because I try to protect people, but they insist and insist. Like me coming in their mouths. And Ijust don't want to say that I'm positive, you know, because I don't want to be [rejected] . . . Let's say I work as a prostitute, so if I say people "no," then it's no business.
}

This participant explained that his concerns about surviving on the streets overshadowed his concern or his ability to attend to the possibility of being infected with HIV.

And so it was kind of like, How can I say this? The possibility was there that I was infected with HIV, but HIV was not killing me today and what was, what was killing me today was, not necessarily killing me, but what was causing me suffering today was, "I don't have a place to live. It's cold. I don't have anywhere to sleep tonight. It's raining."

\title{
DISCUSSION
}

The SNAP study interviews demonstrate the complexity of issues such as communication, intimacy, and HIV prevention strategizing that confront individuals living with HIV. Pledges to avoid transmitting HIV, and expressions of blame or anger toward themselves or their partners, clearly demonstrated participants' attributions of responsibility. Participants also implied their attributions of responsibility while discussing HIV prevention strategies and ideas about communication and decision making. Overall, participants' tendency to attribute responsibility to HIV-negative persons before seroconversion and to HIV-positive persons after seroconversion, signifies a strong sense of personal responsibility both before and after seroconversion. The patterns of attribution after seroconversion are consistent with previous work on self-perceived responsibility in which a majority of HIV-positive participants expressed a sense of personal responsibility for protecting their sex partners (Wolitski \& Bailey, 2005). Our analysis also revealed the intrapersonal, interpersonal, and contextual challenge of maintaining prevention strategies and the emotional impact of being unable to maintain intentions in this regard.

Bimbi and Parsons (2005) examined patterns of attribution of responsibility for HIV infection and found a relationship between attributions and decision making about safer sexual behaviors. One of the four orientations they described was one of "sympathetic orientation" in which HIV-positive individuals attributed responsibility for initial infection to themselves, that is, they retrospectively blamed themselves as 
HIV-negative individuals and then described a sense of responsibility to protect their partners from HIV infection. This orientation was well represented within the interviews analyzed in this study. Although the intention of this analysis was not to associate attributions of responsibility with behavior, the findings do demonstrate that HIV-positive individuals in this study were thinking about the concept of responsibility and that they were willing to talk about their ideas and beliefs.

The complexity of both internal and external factors relating to the HIV prevention strategies of people living with HIV is quite clear in these findings and the concept of personal responsibility is no less complicated. The tendency for HIV-positive men, in these interviews, to claim a strong role of responsibility for HIV prevention is not necessarily an indication that this should be the focus of HIV prevention for HIV-positive individuals. To avoid further stigmatization, or "blaming of the victim," it is crucial that HIV prevention programs address the issue of responsibility by acknowledging the strong sense of responsibility among HIV-positive gay and bisexual men and remaining sensitive to the burden that this sense of responsibility brings.

In addition to acknowledging the burden of responsibility that HIV-positive individuals face, programs can work with these individuals to articulate their own experience or burden and identify possible approaches to managing the burden of responsibility with interventions that reduce stress and increase social support. Another suggestion is to include general stress reduction skills such as role-playing various situations that participants identify as stressful. HIV prevention messages that stress the importance of community or social levels of responsibility for HIV prevention in addition to shared responsibility among partners might also serve to support HIV-positive individuals and reduce their sense of burden for HIV prevention. Furthermore, external factors (such as lack of sexual experience, substance abuse, and daily survival) shown in these results to impede the participants' ability to enact feelings of responsibility need to be addressed in order to improve the chances for success of program participants.

There are some possible limitations to the interpretation and significance of these findings. The attributions of responsibility described may be specific to HIV-positive gay and bisexual men and not similar to attributions of other groups of people living with HIV. Additionally, all men in the SNAP study were HIV-positive when they were interviewed. Their views as HIV-negative men, despite their recent seroconversion, were retrospective and may not accurately represent current attributions of HIV-negative gay and bisexual men. Finally, most participants were recruited from ASOs and may not be representative of men who have no contact with services. Involvement in programs within ASOs may have had an influence on participants' ideas about responsibility.

Future studies might assess participants' program experience and messages about responsibility presented in programs as well as conduct seroconversion narrative studies with other populations such as heterosexual men, women, or people whose narratives primarily involve sharing of needles. There is also a need for more research focused specifically on issues of responsibility and that specifically explore associations between responsibility and behavior. In addition, further studies could explore the issue of stress as a possible result of the burden of responsibility. Finally, many external barriers to effective individual-level HIV prevention strategies (such as substance use and lack of sexual experience) surfaced in these narratives indicating the need for additional research to examine ways of addressing these barriers in order to support HIV-positive individuals in successfully enacting their beliefs and ideas about responsibility for HIV prevention. 


\section{REFERENCES}

Bayer, R. (1996). AIDS prevention-Sexual ethics and responsibility. New England Journal of Medicine, 334, 1540-1542.

Bimbi, D.S., \& Parsons, J.T. (2005). Cause and effect: Attributions about becoming HIV-positive and safer-sex decision making among gay and bisexual men. In P. N. Halkitis, C. A. Gómez, \& R. J. Wolitski (Eds.), HIV+ sex: The psychological and interpersonal dynamics of $H I V$-seropositive gay and bisexual men's relationships (pp. 133-145). Washington, DC: American Psychological Association.

Bingman, C. R., Marks, G., \& Crepaz, N. (2001). Attributions about one's HIV infection and unsafe sex in seropositive men who have sex with men. AIDS and Behavior, 5, 283-289.

Centers for Disease Control and Prevention. (2003). Advancing HIV prevention: New strategies for a changing epidemic-United States, 2003. Morbidity and Mortality Weekly Report, 52, 329-332.

Chen, S. Y., Gibson, S., Katz, M. H., Klausner, J. D., Dilley, J. W., Schwarcz, S. K., et al. (2002). Continuing increases in sexual risk behavior and sexually transmitted diseases among men who have sex with men: San Francisco, Calif, 1999-2001. American Journal of Public Health, 92, 1387-1388.

Colfax, G. N., Mansergh, G., \& Guzman, R., Vittinghoff, E., Marks, G., Rader, M., et al. (2001). Drug use and sexual risk behavior among gay and bisexual men who attend circuit parties: A venue-based comparison. Journal of Acquired Immune Deficiency Syndrome, 28, 373-379.

Crepaz, N., Hart, T. A., \& Marks, G. (2004). Highly active antiretroviral therapy and sexual risk behavior: A meta-analytic review. Journal of the American Medical Association, 292, 224-236.

Crepaz, N., Lyles, C.M., Wolitski, R.J., Passin, W.F., Rama, S.M., Herbst, J.H., et al. (2006). Do prevention interventions reduce HIV risk behaviours among people living with HIV? A meta-analytic review of controlled trials. AIDS, 20, 143-157.

Crepaz, N., \& Marks, G. (2002). Towards an understanding of sexual risk behavior in people living with HIV: A review of social, psychological, and medical findings. AIDS, 16, 135-149.

Gordon, C. M., Forsyth, A. D., Stall, R., \& Cheever, L. W. (2005). Prevention interventions with persons living with HIV/AIDS: State of the science and future directions. AIDS Education and Prevention, 17, 6-20.

Gore-Felton, C., Rotheram-Borus, M.J., Weinhardt, L. S., Kelly, J.A., Lightfoot, M., Kirschenbaum, S.B., et al. (2005). The healthy living project: An individually tailored, multidimensional intervention for HIV-infected persons. AIDS Education and Prevention, 17, 21-39.

Grinstead, O. (2006). Seroconversion narratives and insights for HIV prevention. Focus: A Guide to AIDS Research and Counseling, 21, 1-4.

Grinstead, O., Alvarado, N., Goldstein, E., Woods, W., \& Euren, J. (2005). Seroconversion Narratives for AIDS Prevention: The SNAP Project. (Science to Community Report No. 13). University of California San Francisco, Center for AIDS Prevention Studies.

Johnson, B.T., Carey, M.P., Chaudoir, S.R., \& Reid, A.E. (2006). Sexual risk reduction for persons living with HIV: Research synthesis of randomized controlled trials, 1993 to 2004. Journal of Acquired Immune Deficiency Syndrome, 41, 642-650.

Kok, G. (1999). Targeted prevention for people with HIV/AIDS: Feasible and desirable? Patient Education and Counseling, 36, 239-246.

Marks, G., Bingman, C. R., \& Duval, T. S. (1998). Negative affect and unsafe sex in HIV-positive men. AIDS and Behavior, 2, 89-99.

Marks, G., Burris, S., \& Peterman, T. A. (1999). Reducing sexual transmission of HIV from those who know they are infected: The need for personal and collective responsibility. AIDS, 13, 297-306

National Association of People Living With AIDS. (2005). Principles of HIV Prevention with Positives. Retrieved January 27, 2006, from http://www.napwa.org/documents/ PositivePrevention.pdf

Parsons, J. T., Halkitis, P. N., Wolitski, R. J., Gómez, C. A., \& the Seropositive Urban Men's Study Team. (2003). Correlates of sexual risk behaviors among HIV-positive men who have sex with men. AIDS Education and Prevention, 15, 383-400.

Weinhardt, L.S, Kelly, J.A., Brondino, M.J., Rotheram-Borus, M.J., Kirshenbaum, S.B., Chesney, M.A., et al. (2004). HIV transmission risk behavior among men and women living with HIV in four U.S. cities. Journal of Acquired Immune Deficiency Syndrome, 36, 1057-1066.

Wolitski, R. J., \& Bailey, C. J. (2005). It takes two to tango: HIV-positive gay and bisexual men's beliefs about their responsibility to protect others from HIV infection. In P. N. Halkitis, C. A. Gómez, \& R. J. Wolitski (Eds.), HIV+ sex: The psychological and interpersonal dynamics of HIV-seropositive gay and bisexual men's relationships (pp. 147-162). Washington, DC: American Psychological Association. 